\title{
Access to Mental Health and Substance Use Resources for 2SLGBTQ+ Youth during the COVID-19 Pandemic
}

\author{
Michael Chaiton 1,2,*, Iman Musani ${ }^{1}$, Mari Pullman ${ }^{2}$, Carmen H. Logie ${ }^{3}$, Alex Abramovich ${ }^{1,2,4}(\mathbb{D}$, \\ Daniel Grace ${ }^{1}$ (D) Robert Schwartz ${ }^{1}$ and Bruce Baskerville ${ }^{5,6}$ (D)
}

1 Dalla Lana School of Public Health, University of Toronto, Toronto, ON M5T 3M7, Canada; Iman.musani@mail.utoronto.ca (I.M.); Alex.Abramovich@camh.ca (A.A.); daniel.grace@utoronto.ca (D.G.); Robert.Schwartz@utoronto.ca (R.S.)

2 Institute for Mental Health Policy Research, Centre for Addiction and Mental Health, Toronto, ON M6J 1H4, Canada; mari.pullman@camh.ca

3 Factor-Inwentash Faculty of Social Work, University of Toronto, Toronto, ON M5S 1A1, Canada; carmen.logie@utoronto.ca

4 Department of Psychiatry, University of Toronto, Toronto, ON M5S 1A1, Canada

5 Canadian Institutes of Health Research, Ottawa, ON K1A 0W9, Canada; Bruce.Baskerville@cihr-irsc.gc.ca

6 School of Pharmacy, Faculty of Science, University of Waterloo, Kitchener, ON N2G 1C5, Canada

* Correspondence: Michael.chaiton@utoronto.ca

Citation: Chaiton, M.; Musani, I.;

Pullman, M.; Logie, C.H.;

Abramovich, A.; Grace, D.; Schwartz R.; Baskerville, B. Access to Mental Health and Substance Use Resources for 2SLGBTQ+ Youth during the COVID-19 Pandemic. Int. I. Environ. Res. Public Health 2021, 18, 11315. https://doi.org/10.3390/ ijerph182111315

Academic Editors: Pablo A. Cantero Garlito, Sabina Barrios Fernández, Rodrigo J. Carcedo González and José Carmelo Adsuar Sala

Received: 14 September 2021

Accepted: 25 October 2021

Published: 28 October 2021

Publisher's Note: MDPI stays neutral with regard to jurisdictional claims in published maps and institutional affiliations.

Copyright: (C) 2021 by the authors Licensee MDPI, Basel, Switzerland. This article is an open access article distributed under the terms and conditions of the Creative Commons Attribution (CC BY) license (https:/ creativecommons.org/licenses/by/ $4.0 /)$

\begin{abstract}
Previous research has established that gender and sexual minority (2SLGBTQ+) youth experience worse mental health and substance use outcomes than their heterosexual and cisgender counterparts. Research suggests that mental health and substance use concerns have been exacerbated by the COVID-19 pandemic. The current study used self-reported online survey responses from 1404 Canadian 2SLGBTQ+ youth which included, but were not limited to, questions regarding previous mental health experiences, diagnoses, and substance use. Additional questions assessed whether participants had expressed a need for mental health and/or substance use resources since the beginning of the COVID-19 pandemic (March 2020) and whether they had experienced barriers when accessing this care. Bivariate and multinomial logistic regression analyses were conducted to determine associations between variables and expressing a need for resources as well as experiencing barriers to accessing these resources. Bivariate analyses revealed multiple sociodemographic, mental health, and substance use variables significantly associated with both expressing a need for and experiencing barriers to care. Multinomial regression analysis revealed gender identity, sexual orientation, ethnicity, and level of educational attainment to be significantly correlated with both cases. This study supports growing research on the mental health-related harms that have been experienced during the COVID-19 pandemic and could be used to inform tailored intervention plans for the 2SLGBTQ+ youth population.
\end{abstract}

Keywords: 2SLGBTQ+; COVID-19; youth; pandemic; mental health; substance use; gender minority; sexual minority

\section{Introduction}

The COVID-19 pandemic has had enormous mental health impacts on populations worldwide, with some groups being more vulnerable to poor mental health outcomes than others [1]. Previous research has established that youth who identify as Two-Spirit, lesbian, gay, bisexual, transgender, queer, and questioning (2SLGBTQ+), hereafter also referred to as youth belonging to gender and sexual minority groups, experience mental health concerns at a disproportionately higher rate than their cisgender and/or heterosexual counterparts [2]. While any individual seeking mental health resources may experience general barriers to access, gender and sexual minority individuals often experience additional barriers, which are impediments to care due to stigma against these individuals $[3,4]$. 
Alarmingly, recent research has found gender and sexual minority youth may disproportionately experience mental health-related harms as a result of the COVID-19 pandemic, including heightened anxiety, depression, substance misuse, and suicidality $[1,5,6]$. In a retrospective case series examining psychiatric hospitalizations stemming from the COVID19 pandemic in Los Angeles, California, LGBTQ2S+ youth who were hospitalized for mental health concerns stated that lack of family acceptance during shelter-in-place orders significantly impacted their mental well-being [6]. These sentiments were echoed in another study that was conducted through $Q$ Chat Space, an online professionally facilitated support group center for gender and sexual minority youth operating out of the United States. [7] These youth cited lack of support and inability to utilize established coping mechanisms previously provided by the health care system as reasons for declining mental health during the initial wave of the COVID-19 pandemic [7].

In addition to experiencing greater mental health challenges, there is increasing concern about the ability for 2SLGBTQ+ youth to access quality mental health care during the ongoing COVID-19 pandemic. A report published by the Trevor Project highlighted the immense barriers faced by 2SLGBTQ+ youth in accessing appropriate mental health services in the United States [8]. Existing barriers to access for gender and sexual minority youth include medical mistrust and discrimination from frontline providers. As such, 2SLGBTQ+ youth consistently report lower rates of health care engagement [9]. These barriers have largely been exacerbated in the face of the current pandemic. One explanation for this poorer access is an increased burden on all aspects of the health care system due to COVID-19. As a result of this heightened demand, health care priorities have shifted, leaving children and youth to be deprioritized in many cases [10]. Along with this shift in priorities, stay-at-home orders and physical distancing guidelines have impacted day-today functioning in schools and facilities providing social services $[5,11]$. Research suggests that schools are among the most prominent mental health support providers for 2SLGBTQ+ youth, due in part to stigma many of these youth face in their home environments as well as additional barriers to access for external resources [11]. In-school resources typically include counselling, gender and sexuality alliances, and social supports (e.g., peers, coaches, and teachers) [11]. In the absence of these resources and the inability of schools to provide consistent, in-person support, youth are left without the mental health support they may have previously relied on [5,11].

While there has been a substantial increase in the provision of virtual telehealth services for mental health, 2SLGBTQ+ youth continue to face challenges accessing these services. In a recent study investigating mental health services in the context of COVID-19, 2SLGBTQ+ participants were less than half as likely to utilize telehealth mental health services compared to their heterosexual counterparts [9]. There are several proposed reasons for this underutilization of resources and decreased access to care. In many instances, youth who live in unsupportive home environments have reported feeling unsafe using phone-based services, due to fear of being overheard by family members [7,9]. These youth have also described the feeling of being isolated from their "chosen families" and community supports during the pandemic, an important aspect of support they previously received. They noted that this aspect of peer and community support has not yet been translated to the telehealth services they were being offered. Through testimonials from 2SLGBTQ+ youth, the importance of text-based resources and integrated peer support during the COVID-19 pandemic were heavily emphasized [7].

There have also been limitations noted regarding the nature of care that can be provided through virtual platforms. Notably, for transgender and non-binary youth who are seeking gender-affirming care and treatments (i.e., hormone therapy, transition-related surgeries), there have been considerable disruptions in the access to services [11]. Transgender and non-binary youth have reported increased gender dysphoria as a direct result of being unable to access the necessary gender-affirming treatments [11]. Gender dysphoria has several negative mental health implications, including heightened depression and suicidality $[12,13]$. 
Finally, 2SLGBTQ+ youth who belong to other marginalized groups (i.e., those from lower socioeconomic strata, youth experiencing homelessness) are additionally disadvantaged in their access to care because they are likely to face challenges accessing the reliable, private technology and internet connection necessary to effectively utilize telehealth services [14]. These youth also face additional challenges in relation to COVID-19, including high susceptibility to the virus due to dense living and working conditions, as well as lack of access to other medical supports, including treatments for substance use disorders (e.g., methadone treatments, safe consumption sites) and sexually transmitted infections [14]. These challenges may have impacts on the mental health of vulnerable 2SLGBTQ+ youth and create additional barriers in their ability to access mental health supports during this time.

The current analysis sought to further understand the barriers faced by 2SLGBTQ+ youth to accessing mental health and addiction services during the COVID-19 pandemic. We also aimed to reveal common characteristics of youth who reported experiencing barriers to accessing services with the goal of informing appropriate and accessible services.

\section{Materials and Methods}

\subsection{Data Source}

Data for this analysis were taken from the LGBTQI2S Tobacco Project Screening Questionnaire which surveyed youth between November 2020 and March 2021. Ethics approval for the current study was granted by the University of Toronto. Participants were recruited using paid advertisements through Facebook, Instagram, and Grindr. Additionally, individuals within the networks of youth working group members, advisory group members, and young adult advisors for the project were also recruited through snowball sampling. Investigators used a quota sampling protocol with the goal of the final sample including 900 tobacco smokers and 600 non-smokers. Half of the participants were recruited from three pilot communities: Toronto, Thunder Bay, and Montreal, while the other half were recruited from across Ontario and Quebec; the survey was provided in both English and French. Informed consent was collected online prior to participation in this study. A total of 1500 youth completed the survey and were compensated \$10 CAD each for their participation. Descriptive statistics were calculated for all variables included in the analysis.

\subsection{Variables}

The outcome measure for this analysis was the self-reported experience of barriers to access or delay in accessing mental health or addiction services since March 2020, based on questions developed by Burgess from the Behavioural Risk Factor Surveillance System [15]. The outcome variable was binary and comprised of (1) youth who expressed a need for mental health or addiction services since March 2020 and experienced barriers or delays in accessing these services and (2) youth who expressed a need for mental health or addiction services since March 2020 and did not experience barriers or delays in accessing these services. Individuals were classed based on questions (1) "Since March 2020, was there a time when you wanted to talk with or seek help from a health professional about stress, depression, problems with emotions or substance use?" and (2) "Did you delay or not get the care you thought you needed?" Seeking help from a health professional would include any and all forms of aid including telehealth services. Participants who responded "yes" to the first question and "yes" to the second were classed as having expressed a need for resources and experienced barriers. Those who responded "yes" to the first question and "no" to the second were classed as having expressed a need for resources and not experienced barriers. Individuals who answered "no" to the first question were those who did not express a need for resources to begin with. Additionally, both questions allowed individuals to state "I don't know" or "Prefer not to answer".

Sociodemographic variables included in the analysis were language the survey was conducted in, level of educational attainment, time of residence in Canada (years), individual income, household income, type of municipality (rural/urban), age (years), gender identity, current sexual orientation, and ethnicity. 
Mental health variables included were a lifetime diagnosis of any mental illness (yes/no), current experience of depression (assessed by the short form of the Centre for Epidemiological Studies Depression (CES-D) scale embedded into the survey) [16], suicidal ideation in the past year, and diagnoses of any of 15 individual mental health conditions in a "yes or no" format (anorexia or bulimia, anxiety disorder, attention deficit disorder (ADD), attention deficit hyperactivity disorder (ADHD), bipolar disorder, depression, dysthymia, mania, obsessive compulsive disorder (OCD), panic disorder, phobia, psychosis, posttraumatic stress disorder (PTSD), schizophrenia, and any mental disorder not captured by previous categories).

Substance use variables were also explored as frequency of use in the past year. Substance variables of interest included alcohol (daily use, 4 times a week, 2-3 times a week, once a week, 2-3 times a month, once a month, less than once a month, or not at all), cannabis (same frequency measures as alcohol), cigarette smoking (daily or almost daily, less than daily but at least weekly, less than weekly but at least monthly, less than monthly, or not at all), e-cigarette use (same frequency measures as cigarette smoking), and illicit drug use (any use in the past year). The illicit drugs included in this analysis were poppers/amyl, ketamine, ecstasy/MDMA, crystal meth, crack, cocaine, heroin, other prescription opioids (e.g., Percocet, Dialudid, OxyContin), fentanyl, GHB, tranquilizers or benzodiazepines, psychedelics, and any other illicit drug not captured by the aforementioned categories.

In addition to these sociodemographic, mental health, and substance-related variables, we included participants' self-reported ratings of their physical and mental health. These measures were collected separately in the survey and were measured using a 5-point Likert scale from "Poor" to "Excellent".

\subsection{Bivariate Analysis}

Bivariate analyses were conducted between the outcome variable and each demographic, mental health, and substance use variable included in the sample. For categorical variables of interest, chi square analyses were conducted as cross-tabulations with the outcome variable of barriers to access. For continuous variables in the sample, analyses of variance (ANOVA) were conducted. A significance threshold of $\alpha=0.05$ was used for all bivariate tests. All analyses were conducted using SAS (SAS Institute Inc., Cary, NC, USA) [17] and Stata (StataCorp, College Station, TX, USA) [18].

\subsection{Regression Analyses}

A multinomial logistic regression was conducted on the demographic variables in predicting barriers to accessing mental health/substance use care. The multinomial logistic regression was used to find distinct demographic correlates for individuals who either (a), did not express a need for resources, (b), did express a need for resources and did not face barriers, and (c), did express a need for resources and did face barriers. Again, a significance threshold of $\alpha=0.05$ was used for the logistic regression analyses and $95 \%$ confidence intervals are outlined as well.

\section{Results}

\subsection{Descriptive Statistics}

Descriptive statistics were calculated for sociodemographic, mental health, and substance use variables included in the analysis. Of the original 1500 individuals surveyed, 96 respondents either stated "I don't know" or "Prefer not to answer" or did not provide a response to one or both of the two questions that were used to assess the outcome measures noted above. These individuals were excluded from subsequent analyses and data from 1404 participants were analyzed. A majority of survey respondents reported experiencing a need for mental health/addiction services in the past year and facing barriers when accessing them $(n=815 ; 58.1 \%) .21 .4 \%(n=300)$ of respondents reported experiencing a need for mental health/addiction services and being able to access these resources without 
barriers. Finally, $20.6 \%(n=289)$ stated they did not experience a need for any mental health/addiction services within the past year.

The average age of participants included in this analysis was 21.9 years $(S D=3.78)$, ranging from ages 15 to 29 years. The majority of respondents opted to conduct the survey in English $(n=1142,81.3 \%)$, were White $(n=996,74.3 \%)$ and had resided in Canada for their entire lives $(n=1149,85.9 \%)$. In terms of educational attainment, $34.6 \%$ of respondents $(n=459)$ had a university degree or higher. Participants reported a range of gender identities, $47.1 \%$ of respondents identified their current gender as women $(n=643)$, $23.7 \%$ identified as men $(n=323), 28.4 \%(n=388)$ of these youth identified with gender expansive identities (e.g., gender non-conforming, non-binary, genderqueer, genderfluid) and $0.7 \%(n=10)$ identified as Two-Spirit. Participants also identified with a range of sexual orientations, $29.9 \%$ identified as bisexual $(n=420), 16.7 \%$ identified as queer $(n=234)$, $16.6 \%$ as lesbian $(n=233), 16.0 \%$ as gay $(n=225), 12.6 \%$ as pansexual $(n=177), 4.6 \%$ as asexual $(n=65), 1.1 \%$ as heteroflexible $(n=16), 0.5 \%$ as Two-Spirit $(n=7), 0.4 \%$ as straight/heterosexual $(n=6)$, and $1.5 \%$ of individuals stated that they were unsure or questioning $(n=21)$. Descriptive statistics and bivariate analyses for all demographic variables analyzed can be found in Table 1 . In all following tables, 1-5, categories in which the total sample number does not equal 1404 participants is due to individuals selecting "I don't know" or "Prefer not to answer" in response or leaving the variable measure blank.

Table 1. Cross-Tabulations of Demographic Variables.

\begin{tabular}{|c|c|c|c|c|c|c|c|}
\hline Variable & & $N$ & $\begin{array}{l}\text { Expressed } \\
\text { Need for } \\
\text { Help, Faced } \\
\text { Barriers (\%) }\end{array}$ & $\begin{array}{c}\text { Expressed Need } \\
\text { for Help, Did } \\
\text { Not Face } \\
\text { Barriers (\%) }\end{array}$ & $\begin{array}{l}\text { Did Not } \\
\text { Seek Help } \\
\quad(\%)\end{array}$ & Total $(\%)$ & $p$ \\
\hline Age & & 1404 & 58.1 & 21.4 & 20.6 & $100 \%$ & 0.002 \\
\hline \multicolumn{8}{|l|}{$\begin{array}{l}(M=21.9 \\
S D=3.78)\end{array}$} \\
\hline \multirow[t]{2}{*}{ Language } & English & 1142 & 84.5 & 78.0 & 75.8 & 81.3 & 0.001 \\
\hline & French & 262 & 15.5 & 22.0 & 24.2 & 18.7 & \\
\hline \multirow[t]{6}{*}{ Gender Identity } & Genderfluid & 53 & 4.2 & 4.8 & 2.1 & 3.9 & $<0.001$ \\
\hline & Genderqueer & 62 & 4.2 & 5.8 & 4.2 & 4.5 & \\
\hline & $\begin{array}{c}\text { Gender } \\
\text { non-binary }\end{array}$ & 225 & 18.5 & 19.9 & 7.4 & 16.5 & \\
\hline & $\begin{array}{c}\text { Gender } \\
\text { non-conforming } \\
\text { /Two-Spirit }\end{array}$ & 58 & 4.6 & 5.1 & 2.5 & 4.3 & \\
\hline & Man & 323 & 19.4 & 19.9 & 39.6 & 23.7 & \\
\hline & Woman & 643 & 49.2 & 44.5 & 44.2 & 47.1 & \\
\hline \multirow[t]{8}{*}{ Orientation } & Asexual & 65 & 4.0 & 5.7 & 5.2 & 4.6 & $<0.001$ \\
\hline & Bisexual & 420 & 31.9 & 30.7 & 23.5 & 29.9 & \\
\hline & Gay & 225 & 11.4 & 13.0 & 32.2 & 16.0 & \\
\hline & $\begin{array}{c}\text { Straight/ } \\
\text { Heterosexual/ } \\
\text { Heteroflexible/ } \\
\text { Two-Spirit }\end{array}$ & 29 & 2.1 & 2.7 & 1.7 & 2.1 & \\
\hline & Lesbian & 233 & 16.7 & 13.0 & 20.1 & 16.6 & \\
\hline & Pansexual & 177 & 13.7 & 16.0 & 5.9 & 12.6 & \\
\hline & Queer & 234 & 18.8 & 18.3 & 9.0 & 16.7 & \\
\hline & $\begin{array}{c}\text { Not sure/ } \\
\text { Questioning }\end{array}$ & 21 & 1.5 & 0.7 & 2.4 & 1.5 & \\
\hline
\end{tabular}


Table 1. Cont.

\begin{tabular}{|c|c|c|c|c|c|c|c|}
\hline Variable & & $N$ & $\begin{array}{c}\text { Expressed } \\
\text { Need for } \\
\text { Help, Faced } \\
\text { Barriers (\%) }\end{array}$ & $\begin{array}{c}\text { Expressed Need } \\
\text { for Help, Did } \\
\text { Not Face } \\
\text { Barriers (\%) }\end{array}$ & $\begin{array}{l}\text { Did Not } \\
\text { Seek Help } \\
\quad(\%)\end{array}$ & Total $(\%)$ & $p$ \\
\hline \multirow[t]{10}{*}{ Ethnicity } & East Asian & 48 & 3.4 & 2.5 & 5.1 & 3.6 & \multirow[t]{10}{*}{$<0.001$} \\
\hline & South Asian & 51 & 2.8 & 4.9 & 5.5 & 3.8 & \\
\hline & Southeast Asian & 26 & 2.6 & 1.1 & 1.1 & 1.9 & \\
\hline & $\begin{array}{c}\text { Black- } \\
\text { African/ } \\
\text { Caribbean/ } \\
\text { North American }\end{array}$ & 38 & 2.0 & 2.1 & 5.9 & 2.8 & \\
\hline & $\begin{array}{l}\text { First Nations/ } \\
\text { Indigenous/ } \\
\text { Inuit/Metis }\end{array}$ & 35 & 2.9 & 1.8 & 2.6 & 2.6 & \\
\hline & Latin American & 32 & 2.2 & 2.1 & 3.3 & 2.4 & \\
\hline & Middle Eastern & 27 & 2.4 & 1.8 & 1.1 & 2.0 & \\
\hline & $\begin{array}{c}\text { White-North } \\
\text { American }\end{array}$ & 686 & 49.2 & 50.7 & 57.5 & 51.2 & \\
\hline & White-Other & 310 & 25.3 & 25.0 & 15.0 & 23.1 & \\
\hline & Mixed heritage & 87 & 7.2 & 8.1 & 2.9 & 6.5 & \\
\hline \multirow[t]{4}{*}{ Environment } & $\begin{array}{c}\text { Urban } \\
(100,000+\text { people })\end{array}$ & 892 & 66.9 & 69.6 & 62.2 & 66.5 & \multirow[t]{4}{*}{0.187} \\
\hline & $\begin{array}{c}\text { Medium } \\
\text { city/town } \\
(30,000-99,999 \\
\text { people })\end{array}$ & 279 & 19.3 & 19.9 & 25.8 & 20.8 & \\
\hline & $\begin{array}{c}\text { Small city/town } \\
(1000-29,999 \\
\text { people })\end{array}$ & 131 & 10.6 & 7.3 & 9.9 & 9.8 & \\
\hline & $\begin{array}{c}\text { Rural }(<1000 \\
\text { people })\end{array}$ & 40 & 3.2 & 3.1 & 2.1 & 3.0 & \\
\hline \multirow[t]{6}{*}{ Education } & $\begin{array}{l}\text { Less than high } \\
\text { school }\end{array}$ & 161 & 14.1 & 9.8 & 9.1 & 12.1 & \multirow[t]{6}{*}{$<0.001$} \\
\hline & $\begin{array}{l}\text { High school } \\
\text { diploma }\end{array}$ & 227 & 19.1 & 13.7 & 15.2 & 17.1 & \\
\hline & $\begin{array}{c}\text { Some } \\
\text { post-secondary }\end{array}$ & 250 & 20.5 & 18.9 & 14.1 & 18.8 & \\
\hline & $\begin{array}{l}\text { Registered } \\
\text { apprentice- } \\
\text { ship/trades } \\
\text { certificate or } \\
\text { diploma }\end{array}$ & 18 & 1 & 0.7 & 2.9 & 1.4 & \\
\hline & $\begin{array}{c}\text { College, CEGEP, } \\
\text { other }\end{array}$ & 212 & 16.2 & 18.2 & 13 & 16.0 & \\
\hline & $\begin{array}{l}\text { University } \\
\text { degree }\end{array}$ & 459 & 29.1 & 38.6 & 45.7 & 34.6 & \\
\hline \multirow[t]{2}{*}{$\begin{array}{l}\text { Individual } \\
\text { Income }\end{array}$} & Less than $\$ 15,000$ & 648 & 60.9 & 54.1 & 35.5 & 54.2 & \multirow[t]{2}{*}{$<0.001$} \\
\hline & $\begin{array}{l}\$ 15,000 \text { to } \\
\$ 29,000\end{array}$ & 260 & 20.8 & 25.3 & 20.8 & 21.8 & \\
\hline
\end{tabular}


Table 1. Cont.

\begin{tabular}{|c|c|c|c|c|c|c|c|}
\hline Variable & & $N$ & $\begin{array}{c}\text { Expressed } \\
\text { Need for } \\
\text { Help, Faced } \\
\text { Barriers (\%) }\end{array}$ & $\begin{array}{c}\text { Expressed Need } \\
\text { for Help, Did } \\
\text { Not Face } \\
\text { Barriers (\%) }\end{array}$ & $\begin{array}{l}\text { Did Not } \\
\text { Seek Help } \\
\quad(\%)\end{array}$ & Total (\%) & $p$ \\
\hline & $\begin{array}{l}\$ 30,000 \text { to } \\
\$ 59,999\end{array}$ & 186 & 14.1 & 16 & 19.2 & 15.6 & \\
\hline & $\begin{array}{c}\$ 60,000 \text { to } \\
\$ 79,000\end{array}$ & 75 & 3.2 & 2.3 & 19.2 & 6.3 & \\
\hline & $\begin{array}{l}\text { More than } \\
\$ 80,000\end{array}$ & 26 & 1 & 2.3 & 5.3 & 2.2 & \\
\hline \multirow[t]{6}{*}{$\begin{array}{l}\text { Household } \\
\text { Income }\end{array}$} & Less than $\$ 15,000$ & 131 & 13.9 & 14.7 & 7.6 & 12.7 & $<0.001$ \\
\hline & $\begin{array}{l}\$ 15,000 \text { to } \\
\$ 29,000\end{array}$ & 177 & 18.8 & 18.4 & 11.6 & 17.2 & \\
\hline & $\begin{array}{c}\$ 30,000 \text { to } \\
\$ 59,999\end{array}$ & 221 & 23.4 & 21.7 & 16 & 21.4 & \\
\hline & $\begin{array}{c}\$ 60,000 \text { to } \\
\$ 79,000\end{array}$ & 174 & 13.2 & 13.8 & 29.3 & 16.9 & \\
\hline & $\begin{array}{l}\$ 80,000 \text { to } \\
\$ 100,000\end{array}$ & 118 & 10 & 8.3 & 18.2 & 11.4 & \\
\hline & $\begin{array}{l}\text { More than } \\
\$ 100,000\end{array}$ & 210 & 20.5 & 23 & 17.3 & 20.4 & \\
\hline \multirow[t]{5}{*}{$\begin{array}{l}\text { Length of time in } \\
\text { Canada }\end{array}$} & 2 years or less & 39 & 2.6 & 3.5 & 3.2 & 2.9 & 0.551 \\
\hline & 3 to 5 years & 36 & 2.5 & 1.7 & 4.3 & 2.7 & \\
\hline & 6 to 10 years & 44 & 3.2 & 3.1 & 3.6 & 3.3 & \\
\hline & 11 to 20 years & 70 & 5.3 & 3.8 & 6.4 & 5.2 & \\
\hline & All my life & 1,149 & 86.4 & 87.8 & 82.5 & 85.9 & \\
\hline Total & & 1404 & 58.1 & 21.4 & 20.6 & $100 \%$ & \\
\hline
\end{tabular}

\subsection{Bivariate Analysis}

\subsubsection{Demographic Variables}

Of the demographic variables discussed above, age $(p=0.002)$, language in which the survey was conducted $(p=0.001)$, gender identity $(p<0.001)$, sexual orientation $(p<0.001)$, ethnicity $(p<0.001)$, level of educational attainment $(p<0.001)$, and both individual $(p<0.001)$ and household income $(p<0.001)$ were found to be associated with expressing a need for mental health and addiction services. On average, younger individuals, Englishspeaking individuals, and lower-income individuals (both household and individual) were more likely to have sought services. Variables of interest within the scope of the current study and the direction of these associations with respect to expressing a need for and experiencing barriers to accessing mental health and addiction services are further investigated below under the regression analysis.

\subsubsection{Mental Health Variables}

Most $(69.2 \%)$ participants $(n=967)$ reported that their mental health was either poor or fair on a five-rating scale ranging from poor to excellent. Using the same scale measuring for general health, $67.6 \%$ of participants $(n=936)$ reported that their health was either fair or good. $62.3 \%$ of participants $(n=861)$ had reported experiencing suicidal ideation within the past year and $59.9 \%$ reported that they had received a prior mental health diagnosis $(n=797)$. Self-perceived mental health $(p<0.001)$, self-perceived general health $(p<0.001)$, 
suicidal ideation within the past year $(p<0.001)$, and any mental health diagnosis $(p<0.001)$ were found to be significantly associated with expressing a need to access mental health and addiction services all generally in an inverse direction.

Regarding the above-mentioned variables and specific mental health disorders, both descriptive statistics and bivariate analyses can be found in Table $2.8 .8 \%$ of respondents reported an anorexia/bulimia diagnosis within their lifetime $(n=123), 43.4 \%$ reported anxiety disorder $(n=609), 6.7 \%$ reported ADD $(n=94), 11.4 \%$ reported ADHD $(n=160)$, $5.1 \%$ reported bipolar disorder $(n=75), 37.8 \%$ reported depression $(n=531), 2.6 \%$ reported dysthymia $(n=37), 1.4 \%$ reported mania $(n=20), 7.4 \%$ reported OCD $(n=104), 9.5 \%$ reported panic disorder $(n=134), 2.2 \%$ reported a phobia disorder $(n=31), 1.9 \%$ reported psychosis $(n=26), 12.0 \%$ reported PTSD $(n=168), 0.1 \%$ reported schizophrenia $(n=1)$, and $10.8 \%$ of respondents reported another mental health diagnosis that was not one of the options provided $(n=152)$. Among these mental health diagnoses, anorexia or bulimia $(p=0.003)$, anxiety disorder diagnoses $(p<0.001)$, ADD $(p=0.009)$, ADHD $(p<0.001)$, bipolar $(p=0.024)$, depression (diagnosed $(p<0.001)$ and as measured by the embedded CES-D scale $(p<0.001))$, dysthymia $(p=0.022)$, OCD $(p<0.001)$, panic disorder $(p<0.001)$, phobia $(p=0.038)$, PTSD $(p<0.001)$, and other mental health diagnoses $(p<0.001)$ were associated with expressing a need for services. In all significant associations, a mental health diagnosis increased the likelihood of the individual expressing a need for services.

\subsubsection{Substance Use Variables}

Among variables used to measure substance use, $13.3 \%$ of individuals reported daily tobacco use $(n=186), 9.8 \%$ reported vaping daily $(n=138), 2.4 \%$ reported daily alcohol use $(n=33)$, and $12.2 \%$ reported daily cannabis use $(n=171)$. Current tobacco smoking frequency $(p=0.004)$, alcohol use frequency $(p=0.017)$, and cannabis use frequency $(p=0.001)$ were all associated with the experience of barriers to mental health and addiction services. In addition, any use of illicit substances $(p=0.006)$ within the past year was associated with barriers to access. Within the sample, $31.2 \%$ of participants $(n=438)$ reported using some form of illicit substance within the past year. $3.6 \%$ of individuals reported using poppers or amyl $(n=50), 2.7 \%$ reported using ketamine $(n=38), 8.8 \%$ reported using ecstasy or MDMA $(n=123), 1.1 \%$ reported using crystal meth $(n=15), 1.0 \%$ reported using crack $(n=14), 10.2 \%$ reported using cocaine $(n=143), 1.0 \%$ reported using heroin $(n=14), 6.0 \%$ reported using some form of other prescription drug $(\mathrm{n}=84), 1.2 \%$ reported using fentanyl $(n=17), 1.3 \%$ reported using GHBs $(n=18), 6.9 \%$ reported using tranquilizers or benzodiazepines $(n=97), 18.4 \%$ reported using psychedelics $(n=259)$, and $2.8 \%$ reported using some other illicit substance not listed above over the past year $(n=40)$. Specifically, experiencing barriers to accessing mental health and addiction services was associated with the use of tranquilizers/benzodiazepines $(p=0.031)$ and psychedelic drugs $(p<0.001)$. All data on substance use variables and bivariate analysis can be found in Table 3.

\subsection{Regression Analysis}

A multinomial logistic regression was conducted to determine significant distinctions in demographic variables associated with any of the three situations regarding accessing care: (a), those who did not express a need for resources, (b), those who did express a need for resources and did not face barriers when accessing them, and (c), those who did express a need for resources and did face barriers when accessing them. The analysis identified differences in gender identity, sexual orientation, ethnicity, and level of education to be significant correlates for expressing a need for care as well as experiencing barriers to accessing this care. Data from the multinomial logistic regression can be found in Tables 4 and 5 . 
Table 2. Cross-Tabulations of Mental Health Variables.

\begin{tabular}{|c|c|c|c|c|c|c|c|}
\hline Variable & & $N$ & $\begin{array}{c}\text { Expressed } \\
\text { Need for } \\
\text { Help, Faced } \\
\text { Barriers (\%) }\end{array}$ & $\begin{array}{c}\text { Expressed Need } \\
\text { for Help, Did } \\
\text { Not Face } \\
\text { Barriers (\%) }\end{array}$ & $\begin{array}{l}\text { Did Not } \\
\text { Seek Help } \\
\quad(\%)\end{array}$ & Total (\%) & $p$ \\
\hline \multirow[t]{5}{*}{$\begin{array}{l}\text { Self-perceived } \\
\text { mental health }\end{array}$} & Poor & 467 & 40.6 & 34.4 & 12.2 & 33.4 & $<0.001$ \\
\hline & Fair & 500 & 39.5 & 39.1 & 21.9 & 35.8 & \\
\hline & Good & 272 & 16.2 & 19.1 & 29.2 & 19.5 & \\
\hline & Very good & 132 & 3.6 & 6.7 & 28.8 & 9.4 & \\
\hline & Excellent & 26 & 0.1 & 0.7 & 8 & 1.9 & \\
\hline \multirow{5}{*}{$\begin{array}{l}\text { Self-perceived } \\
\text { general health }\end{array}$} & Poor & 122 & 10.7 & 9.2 & 3.2 & 8.8 & $<0.001$ \\
\hline & Fair & 422 & 35.4 & 29.6 & 17.5 & 30.5 & \\
\hline & Good & 514 & 36.4 & 39.8 & 36.5 & 37.1 & \\
\hline & Very good & 262 & 14.9 & 17.0 & 32.3 & 18.9 & \\
\hline & Excellent & 64 & 2.6 & 4.4 & 10.5 & 4.6 & \\
\hline $\begin{array}{c}\text { Suicidal ideation } \\
\text { (any in the past } \\
\text { year) }\end{array}$ & Yes & 861 & 72.7 & 67.7 & 27 & 62.3 & $<0.001$ \\
\hline $\begin{array}{c}\text { Any mental } \\
\text { health diagnosis }\end{array}$ & Yes & 797 & 65.5 & 77.2 & 25.2 & 59.9 & $<0.001$ \\
\hline $\begin{array}{c}\text { Depression } \\
(\mathrm{CES}-\mathrm{D} ; M= \\
20.05, S D=5.16)\end{array}$ & & & & & & & $<0.001$ \\
\hline ADD & Yes & 94 & 7.0 & 9.3 & 3.1 & 6.7 & 0.009 \\
\hline ADHD & Yes & 160 & 13.0 & 14.7 & 3.5 & 11.4 & $<0.001$ \\
\hline Anorexia/bulimia & Yes & 123 & 9.8 & 10.7 & 3.8 & 8.8 & 0.003 \\
\hline Anxiety disorder & Yes & 609 & 49.2 & 55.0 & 14.9 & 43.4 & $<0.001$ \\
\hline Bipolar disorder & Yes & 72 & 5.6 & 6.7 & 2.1 & 5.1 & 0.024 \\
\hline Depression & Yes & 531 & 42.8 & 48.3 & 12.8 & 37.8 & $<0.001$ \\
\hline Dysthymia & Yes & 37 & 2.7 & 4.3 & 0.7 & 2.6 & 0.022 \\
\hline Mania & Yes & 20 & 1.8 & 1.3 & 0.3 & 1.4 & 0.181 \\
\hline OCD & Yes & 104 & 8.3 & 10.7 & 1.4 & 7.4 & $<0.001$ \\
\hline Panic Disorder & Yes & 134 & 11.7 & 9.7 & 3.5 & 9.5 & $<0.001$ \\
\hline Phobia & Yes & 31 & 2.0 & 4.0 & 1.0 & 2.2 & 0.038 \\
\hline Psychosis & Yes & 26 & 1.7 & 3.3 & 0.7 & 1.9 & 0.054 \\
\hline PTSD & Yes & 168 & 13.9 & 14.7 & 3.8 & 12 & $<0.001$ \\
\hline Schizophrenia & Yes & 1 & 0 & 0.3 & 0 & 0.1 & 0.159 \\
\hline $\begin{array}{c}\text { Other mental } \\
\text { health diagnosis }\end{array}$ & Yes & 152 & 11.3 & 17.3 & 2.8 & 10.8 & $<0.001$ \\
\hline Total & & 1404 & 58.1 & 21.4 & 20.6 & $100 \%$ & \\
\hline
\end{tabular}


Table 3. Cross-Tabulations of Substance Use Variables.

\begin{tabular}{|c|c|c|c|c|c|c|c|}
\hline Variable & & $N$ & $\begin{array}{c}\text { Expressed } \\
\text { Need for } \\
\text { Help, Faced } \\
\text { Barriers (\%) }\end{array}$ & $\begin{array}{c}\text { Expressed Need } \\
\text { for Help, Did } \\
\text { Not Face } \\
\text { Barriers (\%) }\end{array}$ & $\begin{array}{l}\text { Did Not } \\
\text { Seek Help } \\
\quad(\%)\end{array}$ & Total (\%) & $p$ \\
\hline \multirow[t]{3}{*}{ Alcohol use } & None & 210 & 13.0 & 14.3 & 21.1 & 15.0 & 0.017 \\
\hline & Daily & 33 & 2.1 & 2.7 & 2.8 & 2.4 & \\
\hline & Less than daily & 1161 & 84.9 & 83.0 & 76.1 & 82.7 & \\
\hline \multirow[t]{3}{*}{ Cannabis use } & None & 512 & 34.2 & 36.0 & 43.3 & 36.5 & 0.001 \\
\hline & Daily & 171 & 14.1 & 13.3 & 5.5 & 12.2 & \\
\hline & Less than daily & 721 & 51.7 & 50.7 & 51.2 & 51.4 & \\
\hline \multirow[t]{3}{*}{ Tobacco use } & None & 927 & 66.1 & 68.3 & 63.8 & 66.1 & 0.004 \\
\hline & Daily & 186 & 12.5 & 9.3 & 19.5 & 13.3 & \\
\hline & Less than daily & 289 & 21.3 & 22.3 & 16.7 & 20.6 & \\
\hline \multirow[t]{3}{*}{ Vaping } & None & 1022 & 72.9 & 74.0 & 71.3 & 72.8 & 0.543 \\
\hline & Daily & 138 & 10.4 & 9.7 & 8.3 & 9.8 & \\
\hline & Less than daily & 244 & 16.7 & 16.3 & 20.4 & 17.4 & \\
\hline $\begin{array}{l}\text { Past year illicit } \\
\text { substance use } \\
\text { (overall) }\end{array}$ & Yes & 438 & 32.6 & 34.7 & 23.5 & 31.2 & 0.006 \\
\hline Past year cocaine & Yes & 143 & 10.4 & 10.3 & 9.3 & 10.2 & 0.867 \\
\hline Past year crack & Yes & 14 & 0.7 & 1.3 & 1.4 & 1.0 & 0.511 \\
\hline $\begin{array}{l}\text { Past year crystal } \\
\text { meth }\end{array}$ & Yes & 15 & 1.1 & 0.3 & 1.7 & 1.1 & 0.254 \\
\hline $\begin{array}{l}\text { Past year } \\
\text { ecstasy/ } \\
\text { MDMA }\end{array}$ & Yes & 123 & 10.1 & 7.3 & 6.6 & 8.8 & 0.121 \\
\hline $\begin{array}{l}\text { Past year } \\
\text { fentanyl }\end{array}$ & Yes & 17 & 1.0 & 0.7 & 2.4 & 1.2 & 0.098 \\
\hline Past year GHB & Yes & 18 & 1.1 & 1.0 & 2.1 & 1.3 & 0.400 \\
\hline Past year heroin & Yes & 14 & 1.0 & 0.3 & 1.7 & 1.0 & 0.233 \\
\hline $\begin{array}{l}\text { Past year } \\
\text { ketamine }\end{array}$ & Yes & 38 & 3.3 & 2.0 & 1.7 & 2.7 & 0.252 \\
\hline $\begin{array}{c}\text { Past year } \\
\text { poppers/amyl }\end{array}$ & Yes & 50 & 3.6 & 5.0 & 2.1 & 3.6 & 0.160 \\
\hline $\begin{array}{l}\text { Past year other } \\
\text { prescription } \\
\text { drugs }\end{array}$ & Yes & 84 & 7.1 & 5.3 & 3.5 & 6.0 & 0.069 \\
\hline $\begin{array}{c}\text { Past year } \\
\text { psychedelics }\end{array}$ & Yes & 259 & 20.2 & 22.0 & 9.7 & 18.4 & $<0.001$ \\
\hline $\begin{array}{l}\text { Past year tran- } \\
\text { quilizers/benzos }\end{array}$ & Yes & 97 & 6.9 & 9.7 & 4.2 & 6.9 & 0.031 \\
\hline $\begin{array}{l}\text { Past year other } \\
\text { substances }\end{array}$ & Yes & 40 & 3.2 & 3.7 & 1.0 & 2.8 & 0.106 \\
\hline Total & & 1404 & 58.1 & 21.4 & 20.6 & $100 \%$ & \\
\hline
\end{tabular}


Table 4. Redictors of seeking help for mental health and substance use issues without barriers or delays to access among 2SLGBTQ+ youth and youth adults (16-29) Canada, 2021.

\begin{tabular}{|c|c|c|c|c|c|}
\hline \multirow{2}{*}{$\begin{array}{c}\text { Variables } \\
\text { Age }\end{array}$} & & \multirow{2}{*}{$\begin{array}{c}\text { Odds Ratio } \\
1\end{array}$} & \multirow{2}{*}{$\frac{p}{0.999}$} & \multicolumn{2}{|c|}{ 95\% Confidence Interval } \\
\hline & & & & 0.944 & 1.06 \\
\hline \multirow[t]{6}{*}{ Gender } & Man & 1 & . & . & . \\
\hline & Genderfluid & 2.189 & 0.153 & 0.748 & 6.402 \\
\hline & Genderqueer & 1.085 & 0.851 & 0.465 & 2.533 \\
\hline & Gender non-binary & 3.075 & $0.001 * *$ & 1.585 & 5.966 \\
\hline & $\begin{array}{c}\text { Gender non-conforming/ } \\
\text { Two-Spirit }\end{array}$ & 2.885 & $0.04 *$ & 1.048 & 7.944 \\
\hline & Woman & 1.611 & 0.045 * & 1.01 & 2.481 \\
\hline \multirow[t]{8}{*}{ Orientation } & Asexual & 1 & & & \\
\hline & Bisexual & 0.572 & 0.136 & 0.272 & 1.193 \\
\hline & Gay & 0.428 & $0.002 * *$ & 0.25 & 0.73 \\
\hline & $\begin{array}{l}\text { Straight/Heterosexual/ } \\
\text { Heteroflexible/Two-Spirit }\end{array}$ & 0.803 & 0.716 & 0.247 & 2.616 \\
\hline & Lesbian & 0.634 & 0.053 & 0.403 & 1.007 \\
\hline & Pansexual & 1.991 & 0.048 * & 1.005 & 3.946 \\
\hline & Queer & 1.609 & 0.1 .5 & 0.906 & 2.857 \\
\hline & Not sure/Questioning & 0.547 & 0.288 & 0.18 & 1.664 \\
\hline \multirow[t]{9}{*}{ Ethnicity } & White & 1 & . & . & . \\
\hline & East Asian & 1.081 & 0.842 & 0.502 & 2.338 \\
\hline & South Asian & 0.439 & $0.042 *$ & 0.198 & 0.971 \\
\hline & Southeast Asian & 2.251 & 0.296 & 0.492 & 10.3 \\
\hline & $\begin{array}{c}\text { Black-African/Caribbean/ } \\
\text { North American }\end{array}$ & 0.454 & 0.051 & 0.206 & 1.002 \\
\hline & $\begin{array}{c}\text { First Nations/ } \\
\text { Indigenous/Inuit/Metis }\end{array}$ & 0.855 & 0.754 & 0.32 & 2.285 \\
\hline & Latin American & 0.517 & 0.171 & 0.201 & 1.328 \\
\hline & Middle Eastern & 2.034 & 0.289 & 0.548 & 7.553 \\
\hline & Mixed heritage & 2.878 & $0.022 *$ & 1.169 & 7.09 \\
\hline $\begin{array}{l}\text { Environment } \\
\text { Population }\end{array}$ & & 0.868 & 0.155 & 0.714 & 1.055 \\
\hline \multirow[t]{6}{*}{ Education } & Less than high school & 1 & & & \\
\hline & High school diploma & 0.766 & 0.392 & 0.416 & 1.411 \\
\hline & Some post-secondary & 0.906 & 0.764 & 0.477 & 1.722 \\
\hline & $\begin{array}{l}\text { Registered apprenticeship/ } \\
\text { trades certificate or diploma }\end{array}$ & 0.391 & 0.165 & 0.104 & 1.469 \\
\hline & College, CEGEP, other & 0.844 & 0.631 & 0.423 & 1.684 \\
\hline & University degree & 0.43 & $0.015^{*}$ & 0.217 & 0.849 \\
\hline $\begin{array}{c}\text { Length of time in } \\
\text { Canada }\end{array}$ & & 1.147 & 0.101 & 0.974 & 1.352 \\
\hline
\end{tabular}


Table 5. Predictors of seeking help for mental health and substance use issues with barriers or delays to access among 2SLGBTQ+ youth and youth adults (16-29) Canada, 2021.

\begin{tabular}{|c|c|c|c|c|c|}
\hline \multirow{2}{*}{$\begin{array}{c}\text { Variables } \\
\text { Age }\end{array}$} & & \multirow{2}{*}{$\frac{\text { Odds Ratio }}{0.999}$} & \multirow{2}{*}{$\frac{p}{0.959}$} & \multicolumn{2}{|c|}{$95 \%$ Confidence Interval } \\
\hline & & & & 0.932 & 1.069 \\
\hline \multirow[t]{6}{*}{ Gender } & Man & 1 & & & \\
\hline & Genderfluid & 2.256 & 0.186 & 0.675 & 7.544 \\
\hline & Genderqueer & 1.322 & 0.584 & 0.488 & 3.582 \\
\hline & Gender non-binary & 3.514 & $0.001 * *$ & 1.656 & 7.455 \\
\hline & $\begin{array}{c}\text { Gender non-conforming/ } \\
\text { Two-Spirit }\end{array}$ & 3.479 & 0.031 * & 1.119 & 10.81 \\
\hline & Woman & 1.436 & 0.229 & 0.796 & 2.59 \\
\hline \multirow[t]{8}{*}{ Orientation } & Asexual & 1 & & & \\
\hline & Bisexual & 0.867 & 0.738 & 0.375 & 2.001 \\
\hline & Gay & 0.438 & 0.014 * & 0.227 & 0.844 \\
\hline & $\begin{array}{l}\text { Straight/Heterosexual/ } \\
\text { Heteroflexible/Two-Spirit }\end{array}$ & 1.288 & 0.704 & 0.349 & 4.76 \\
\hline & Lesbian & 0.521 & $0.024 *$ & 0.296 & 0.918 \\
\hline & Pansexual & 2.222 & 0.037 * & 1.049 & 4.706 \\
\hline & Queer & 1.361 & 0.354 & 0.71 & 2.612 \\
\hline & Not sure/Questioning & 0.153 & 0.091 & 0.017 & 1.352 \\
\hline \multirow[t]{9}{*}{ Ethnicity } & White & 1 & & & \\
\hline & East Asian & 0.648 & 0.42 & 0.226 & 1.861 \\
\hline & South Asian & 0.824 & 0.672 & 0.335 & 2.024 \\
\hline & Southeast Asian & 0.827 & 0.854 & 0.11 & 6.215 \\
\hline & $\begin{array}{c}\text { Black-African/Caribbean/ } \\
\text { North American }\end{array}$ & 0.452 & 0.125 & 0.164 & 1.247 \\
\hline & $\begin{array}{c}\text { First Nations/ } \\
\text { Indigenous/Inuit/Metis }\end{array}$ & 0.516 & 0.318 & 0.141 & 1.892 \\
\hline & Latin American & 0.557 & 0.335 & 0.169 & 1.832 \\
\hline & Middle Eastern & 2.085 & 0.336 & 0.467 & 9.317 \\
\hline & Mixed heritage & 3.864 & $0.006^{* *}$ & 1.482 & 10.075 \\
\hline $\begin{array}{l}\text { Environment } \\
\text { Population }\end{array}$ & & 0.809 & 0.081 & 0.638 & 1.027 \\
\hline \multirow[t]{6}{*}{ Education } & Less than high school & 1 & & & \\
\hline & High school diploma & 0.937 & 0.867 & 0.435 & 2.018 \\
\hline & Some post-secondary & 1.377 & 0.429 & 0.626 & 3.015 \\
\hline & $\begin{array}{l}\text { Registered apprenticeship/ } \\
\text { trades certificate or diploma }\end{array}$ & 0.436 & 0.377 & 0.069 & 2.752 \\
\hline & College, CEGEP, other & 1.648 & 0.244 & 0.711 & 3.82 \\
\hline & University degree & 0.991 & 0.983 & 0.429 & 2.289 \\
\hline $\begin{array}{l}\text { Length of Time in } \\
\text { Canada }\end{array}$ & & 1.154 & 0.155 & 0.947 & 1.406 \\
\hline
\end{tabular}




\subsubsection{Expressing a Need for Care}

In comparing correlates that distinguished individuals who did express a need for care from those who did not, gender, sexual orientation, ethnicity, and education were found to be significant. Certain gender identity and sexual orientation categories were combined to provide greater statistical power. The researchers believed that any variable with less than 20 participants would be too insignificant to hold statistical power in the context of the broader sample. For gender, 10 individuals identified as Two-Spirit and were therefore combined with the next smallest label, gender non-conforming $(n=48)$ to create a new label with a larger sample size $(n=58)$. For sexual orientation, heteroflexible $(n=16)$, straight/heterosexual $(n=6)$, and Two-Spirit $(n=7)$ were combined into one label $(n=29)$ for the same reason.

Those who identified as gender non-binary (OR 3.08, 95\% CI 1.59, 6.00), gender nonconforming/Two-Spirit (OR 2.89, 95\% CI 1.05, 7.94), or as women (OR 1.61, 95\% CI 1.01, 2.48) were significantly more likely to express a need for care than those who identified as men. Those who identified as pansexual (OR 1.99, 95\% CI 1.01, 3.95) were more likely to express a need for care than those who identified their sexual orientation as bisexual. Conversely, those who identified as gay (OR $0.43,95 \%$ CI $0.25,0.73$ ) were significantly less likely to express a need for care than those who identified as bisexual. Furthermore, those who reported to be mixed-race (OR $2.88,95 \%$ CI $1.17,7.09$ ) were more likely than White participants to have expressed a need for care. However, South Asian participants (OR $0.44,95 \%$ CI $0.20,0.97$ ) were less likely than White participants to have expressed this need. Additionally, University educated individuals (OR $0.43,95 \%$ CI $0.22,0.85$ ) were found to be less likely to have expressed a need for care than those who did not complete high school.

\subsubsection{Expressing a Need for Care and Experiencing Barriers}

Comparing participants who had expressed a need for resources and did face barriers when accessing them to those who did not express a need for resources revealed that gender identity, sexual orientation, and ethnicity were significantly associated. Individuals who identified as gender non-binary (OR 3.51, 95\% CI 1.66, 7.46) and gender non-conforming/Two-Spirit (OR 3.48, 95\% CI 1.12, 10.81) reported experiencing barriers to access at a significantly higher rate than those who identified as men. Those who identified as pansexual (OR 2.22, 95\% CI 1.05, 4.71) were significantly more likely to report experiencing barriers to resources than those who identified as bisexual. However, those who identified as gay (OR $0.44,95 \%$ CI $0.23,0.84$ ) and lesbian (OR $0.52,95 \%$ CI $0.30,0.92$ ) were significantly less likely to report experiencing these barriers compared to those who identified as bisexual. Furthermore, mixed-race individuals (OR 3.87, 95\% CI 1.48, 10.08) also reported experiencing barriers to accessing these resources in comparison to White participants.

\subsubsection{Experiencing Barriers to Access}

A post hoc analysis conducted to determine significant distinctions in individuals who did face barriers as opposed to individuals who did not face barriers in accessing care revealed that education was the only significant association. Specifically, those who were college / Collège d'enseignement général et professionnel (CEGEP) educated (or something of equivalent experience) $(\mathrm{OR}=0.51,95 \% \mathrm{CI} 0.26,0.99)$ or university educated $(\mathrm{OR}=0.42$, $95 \%$ CI $0.21,0.48$ ) were significantly less likely than those who were less educated to face barriers when trying to access care.

\section{Discussion}

\subsection{Implications for Research}

The goal of this analysis was to better understand common characteristics of 2SLGBTQ+ youth who have experienced barriers to accessing mental health services in Canada during the COVID-19 pandemic. Our exploratory analysis sought to reveal areas for future study and potential focus areas for mental health services directed at gender and sexual minority 
youth. To date, little research has investigated the barriers to accessing mental health services among this heterogeneous group in a Canadian context [7].

Our results support an emerging body of research suggesting that gender and sexual minority youth are experiencing immense mental health challenges during the current COVID-19 pandemic $[8,11,14]$. Approximately $80 \%$ of youth who responded to the current survey stated that they felt the need to seek mental health and/or addiction supports since March 2020. Further, the majority of respondents (62.3\%) expressed experiencing some form of suicidal ideation in the past year. A study conducted in 2019, prior to the COVID-19 pandemic, reported that $42 \%$ of the gender and sexual minority youth surveyed had accounts of suicidal ideation [19]. Although the 2019 study asked respondents about suicidal ideation within the last six months as opposed to the last year as was asked in the current study, it may still suggest that the COVID-19 pandemic has exacerbated thoughts of suicide. In addition to a high proportion of respondents reporting mental health challenges, our results reinforced research which suggested that gender and sexual minority youth faced substantial barriers to accessing mental health and addiction services during this time, with a majority of respondents stating that they experienced barriers to accessing mental health or addiction services since March $2020[12,20]$. Some of these mental health and substance use impacts may be experienced more broadly, but the survey responses do suggest that the COVID-19 pandemic has served to exacerbate some of these effects.

Our analysis revealed some of the sociodemographic, mental health, and substancerelated factors that vary among youth who express a need for mental health and/or addiction services and are unable to adequately access such services. Notably, we observed that youth who reported facing barriers to access varied significantly based on socioeconomic indicators including income (both household and individual), and education level. This finding is in line with research suggesting that low socioeconomic status is associated with decreased access to mental health services for youth, both within and beyond the context of the COVID-19 pandemic [21,22]. In addition to socioeconomic indicators, we observed that the age of participants was associated with barriers to access. This association should be investigated further, as age often correlates with other important characteristics such as income and educational attainment, especially between the ages of 15 and 29 [23,24].

Multiple sociodemographic variables were found to be significant correlates both for expressing a need for care and experiencing barriers in finding care. Differences in gender identity, sexual orientation, ethnicity, and education were found to be significantly associated in distinguishing individuals who expressed a need for care from those who did not. Furthermore, distinctions in gender identity, sexual orientation, and ethnicity were significant correlates in distinguishing those who experienced barriers when seeking care from those who did not express a need for care.

\subsubsection{Expressing a Need for Care}

Those who identified as gender non-binary, gender non-conforming/Two-Spirit and women were more likely to express a need for care than those who identified as men. This observation may be due to societal pressure to not express a need for mental health resources that men experience, which has been well researched $[25,26]$. Previous research has identified that men are often pressured to conform to outdated gender norms regarding masculinity and may feel that expressing a need or seeking help for mental health opposes this expectation [25]. Individuals who identified as pansexual were more likely to express a need for care than those who identified as bisexual. However, those who identified as gay were less likely to express a need for care than those who identified as bisexual. This pattern may be explained by past research on sexual orientation and the binarity/monosexuality associated with these categories [27]. Individuals who identified with non-monosexual labels, such as bisexual or pansexual, may experience discrimination due to this and may find difficulty assimilating with a group. This may lead to frustration and isolation in youth that affects mental health, leading them to seek resources for help [27]. 
Regarding other sociodemographic factors correlated with expressing a need for care, mixed-race individuals were more likely than White participants to have expressed this need whereas South Asians were less likely to have. Previous studies on racial identity have mirrored the above research on less categorical sexual identities. Mixed-race individuals may struggle with mental health in youth due to added identity frustration because of their mixed heritage which may serve to exacerbate any existing conditions [28]. Furthermore, in a study focusing on American military personnel, the researchers found that amongst all racial groups studied (Asian, White, Black, Indigenous and Other), Asian Americans were the least likely to utilize available mental health resources [29]. This suggests that cultural differences in how expressing a need for and/or seeking mental health resources is perceived may account for South Asians attempting to access these resources at a decreased rate.

Lastly, university educated individuals were less likely than their counterparts who had not completed high school to have expressed a need for these resources. This may be socioeconomic in nature, those who can afford university typically come from wealthier backgrounds which tend to have lower rates of mental illness [30]. Specifically, with regard to the COVID-19 pandemic, factors related to lower education such as scarce job opportunities, especially those in which individuals can work from home, and less access to reliable information regarding COVID-19, may act as an additional stressor exacerbating underlying mental health conditions [31].

\subsubsection{Expressing a Need for Care and Experiencing Barriers}

Distinguishing those who did express a need for care and did face barriers from those who did not express a need for these resources, those who identified as gender nonbinary and gender non-conforming/Two-Spirit reported experiencing barriers to access at a greater rate than those who identified as men. Furthermore, those who identified as pansexual were most likely to have expressed a need for resources followed by those who identified as bisexual, then as gay, similar patterns can be observed in individuals who experience barriers to accessing care. Those who identified as pansexual were much more likely to report experiencing barriers to resources followed by those who identified as bisexual, then those who identified as gay and lesbian. Those who identify with a non-monosexual or non-unigender label may find resources and services to be limited because they have not been expanded to be inclusive and affirming to all individuals within the population [32]. Although mental health and substance use services may be 2SLGBTQ+ affirming and inclusive, they should cater to all individuals within the population and should receive ongoing and mandatory 2SLGBTQ+ inclusion training and education to ensure this.

Furthermore, mixed-race individuals reported experiencing barriers to accessing these resources in comparison to White participants. Similar to resources that may exist that focus on specific gender and sexual minority individuals, those with less categorical or mixed racial identities may find that resources that cater specifically to them are limited. For mixed-race individuals with a blend of cultural identities and influences, they may find difficulty in accessing resources that are inclusive to their identity. Our findings on barriers experienced by those who are sexual and gender diverse and/or of racial minority backgrounds highlights the importance of finding culturally appropriate mental health services.

Lastly, findings revealed that university, college, and CEGEP educated individuals were less likely to experience barriers than those who had not obtained a high school diploma. Again, this may be socioeconomic in nature, while higher educated individuals are less likely to express a need for resources in the first place, when they do, they may have the financial resources to find the appropriate supports without barriers.

Regarding mental health diagnoses, we found that mental health status over the past year (both as a diagnosed mental disorder and as a self-reported current mental health rating) was associated with experiencing barriers to accessing services. When examining 
specific mental health diagnoses, we noted that a majority of individual mental disorder diagnoses were associated with experiencing barriers to accessing services (including, but not limited to, anxiety, depression, anorexia or bulimia, and OCD; full results can be seen in Table 2). This finding supports a strong body of research suggesting that different mental illnesses are associated with varying levels of stigma, social acceptability, and treatment options [33]. Moreover, youth belonging to gender and sexual minority groups face unique experiences of mental health that intersect with their gender and sexual identities $[1,5,10]$. As such, despite an expedited introduction of many telehealth-based services seeking to address mental health concerns for these young people, it is evident that current services may not address the complexity necessary to adequately support gender or sexual minority youth.

In addition to diagnosed mental illness, we observed that any suicidal ideation in the past year was associated with barriers to accessing mental health/addiction services. This finding is of particular concern. Young people who belong to gender and sexual minority groups frequently report suicidal ideation at much higher rates than their cisgender and heterosexual counterparts [34]. A recent study suggested that feelings of community connectedness among 2SLGBTQ+ youth is an important protective factor against suicidal ideation and the progression of poor mental health [34]. The understanding that much of the community connectedness youth were experiencing before the pandemic has been removed due to physical distancing requirements, along with the heightened prevalence of suicidal ideation among these groups in general, points to the need for mental health services to be accessible and sufficient for youth in these groups $[5,7,14,34]$.

Finally, we examined substance use variables and found that frequency of smoking cigarettes, using cannabis, and consuming alcohol were associated with experiencing barriers to accessing mental health/addiction services. Notably, we did not observe any association between frequency of e-cigarette (vaping) use and the experience of barriers to access. The use of legal and widely available substances, such as alcohol, cigarettes, and cannabis, has been found to occur alongside mental health challenges and mental disorders. [35-38] Given this high degree of co-occurrence, it is possible that the relationship between barriers to accessing services and the use of legal substances are due to their association with the presence of a mental illness.

Regarding the use of illicit substances, we found that overall use of illicit substances was found to be associated with the experience of barriers to access, as well as the use of tranquilizers/benzodiazepines and psychedelic substances in the past year. This finding is of concern because these substances have potential to cause a great degree of harm on their own, as well as when they exist alongside other substance use and mental health challenges [39]. In addition to these harms, recent studies have noted that gender or sexual minority youth have reported using substances more often during the COVID-19 pandemic $[11,40]$. The nature of this association between substance use and access to mental health/addiction services must be investigated further.

\subsection{Implications for Practice}

Our findings on suicidal ideation and the need for mental health services within this community are significant and serve as a call to action. Similar findings documenting unmet needs within the 2SLGBTQ+ population and subpopulatins within the community have been previously documented $[41,42]$. The striking figures regarding mental health disruptions experienced by the 2SLGBTQ+ youth population (59.9\% of our population reported a previous mental health diagnosis), especially in comparison to the general youth population in Canada, of which approximately $20 \%$ have a mental health disorder [43], highlights how mental health services must be tailored to where the greatest needs lie. Previous studies that surveyed members of the 2SLGBTQ+ community on where they believe health care services are lacking suggested that sexual minority affirming care needs to be emphasized; many believed that care is often heterocentric and "one-size-fitsall", disregarding the experiences of sexual minority patients $[3,44]$. Service providers 
should recognize that gender and sexual minority individuals may have had previous experiences with health care providers in which they may have been discriminated against or stigmatized; these individuals may continue to feel distrust when approaching service providers and the providers should work to lessen these hesitations [3]. The current study successfully identified that increased mental health disruptions experienced by 2SLGBTQ+ youth can be attributed, at least in some part, to barriers that are experienced when attempting to access the appropriate services. The researchers encourage future studies to identify where these barriers experienced by the 2SLGBTQ+ youth population specifically lie.

\subsection{Limitations}

Although the current study provides valuable insight into the need for mental health and substance use resources experienced by gender and sexual minority youth, there are several limitations that should be mentioned. While the survey was distributed throughout a variety of social spheres, the sample was skewed to represent more White, urban dwelling, and University educated individuals. This may have caused some imbalance in the representation of individuals from other backgrounds within the sample. It should also be noted that there were 22 respondents that identified as straight, heterosexual, or heteroflexible. This is not enough people to make a comparison against the 2SLGBTQ+ respondents within the sample. Therefore, the current study cannot conclude that 2SLGBTQ+ individuals experience mental health and/or substance use related harms and challenges at a greater rate in comparison to their cisgender and heterosexual counterparts, although other studies mentioned above do suggest that this seems evident.

As with any study of this nature, the inability to infer causation from correlational research must be noted. The sociodemographic and mental health/substance use variables associated with experiencing a need for mental health/substance use services and/or experiencing barriers to accessing these services cannot be seen as causal factors. It may be the case that some of these variables are associated with an overarching factor that is more strongly associated with these two cases.

Additionally, although studies have determined that many of the services offered to 2SLGBTQ+ individuals have been limited due to the COVID-19 pandemic, and it can possibly be inferred that this would heighten the need for mental health/substance use care, the current study did not have a baseline assessment that was conducted prior to the beginning of the pandemic. Rather, a retrospective approach was used to assess the need for and barriers to accessing 2SLGBTQ+ youth mental health and/or substance use resources since the beginning of COVID-19 pandemic. Therefore, participant recall regarding these variables may potentially be inaccurate and subject to biases.

\section{Conclusions}

Overall, our findings support ongoing and emerging studies from other regions which have suggested that the COVID-19 pandemic has led to disproportionate mental healthrelated harms for vulnerable groups, including 2SLGBTQ+ youth. Furthermore, the current study revealed that a majority of the respondents not only expressed experiencing a need for mental health and addictions services after March 2020, but also faced barriers when attempting to access them. This analysis provided a valuable basis for future studies in a Canadian context to further investigate commonalities between individuals who are experiencing the mental health impacts of the COVID-19 pandemic. Future studies should clarify the nature of these associations in order to understand and address challenges faced by youth who are especially vulnerable to the immediate and long-term impacts of the current COVID-19 pandemic. 
Author Contributions: Conceptualization, M.C., I.M., M.P., C.H.L., A.A., D.G., R.S. and B.B.; methodology, M.C.; formal analysis, M.C., I.M. and M.P.; writing-original draft preparation, I.M., M.P.; writing-review and editing, M.C., I.M., M.P., C.H.L., A.A., D.G., R.S. and B.B.; funding acquisition, M.C., C.H.L., A.A., D.G., R.S. and B.B. All authors have read and agreed to the published version of the manuscript.

Funding: This research was funded by Canadian Institutes of Health Research.

Institutional Review Board Statement: This study was conducted according to the guidelines of the Declaration of Helsinki, and approved by the Institutional Review Board (or Ethics Committee) of Centre for Addiction and Mental Health (protocol code 158-2020 and 19 February 2021.

Informed Consent Statement: Informed consent was obtained online from all subjects involved in this study.

Data Availability Statement: Anonymized data can be requested from the corresponding author from investigators at academic institutions with research ethics board approval.

Conflicts of Interest: The authors declare no conflict of interest. The funders had no role in the design of this study; in the collection, analyses, or interpretation of data; in the writing of the manuscript, or in the decision to publish the results.

\section{References}

1. Asmundson, G.J.; Blackstock, C.; Bourque, M.C.; Brimacombe, G.; Crawford, A.; Deacon, S.H.; McMullen, K.; McGrath, P.J.; Mushquash, C.; Stewart, S.H.; et al. Easing the disruption of COVID-19: Supporting the mental health of the people of Canada-October 2020_An RSC Policy Briefing. Facets 2020, 5, 1071-1098. [CrossRef]

2. Centers for Disease Control and Prevention. Health Disparities among LGBTQ Youth. Available online: https://www.cdc.gov / healthyyouth/disparities/health-disparities-among-lgbtq-youth.htm (accessed on 28 July 2021).

3. Gaspar, M.; Marshall, Z.; Rodrigues, R.; Adam, B.D.; Brennan, D.J.; Hart, T.A.; Grace, D. Mental health and structural harm: A qualitative study of sexual minority men's experiences of mental healthcare in Toronto, Canada. Cult. Health Sex. 2019, $23,98-114$. [CrossRef] [PubMed]

4. Abramovich, A. Preventing, reducing, and ending LGBTQ2S youth homelessness: The need for targeted strategies. Soc. Incl. 2016, 4, 86-96. [CrossRef]

5. Salerno, J.P.; Williams, N.D.; Gattamorta, K.A.; Kendall-Tackett, K. LGBTQ populations: Psychologically vulnerable communities in the COVID-19 pandemic. Psychol. Trauma 2020, 12, 239-242. [CrossRef] [PubMed]

6. Choi, K.R.; Martinez-Hollingsworth, A.; Mead, M.; Dappolonia, M.S. Adolescent Psychiatric Emergencies Precipitated by the COVID-19 Pandemic. J. Psychosoc. Nurs. Ment. Health Serv. 2021, 59, 17-21. [CrossRef] [PubMed]

7. Fish, J.N.; McInroy, L.B.; Paceley, M.S.; Williams, N.D.; Henderson, S.; Levine, D.S.; Edsall, R.N. “I'm kinda stuck at home with unsupportive parents right now": LGBTQ youths' experiences with COVID-19 and the importance of online support. J. Adolesc. Health 2020, 67, 450-452. [CrossRef] [PubMed]

8. The Trevor Project; Green, A.; Dorison, S.; Price-Feeny, M. Implications of COVID-19 for LGBTQ Youth Mental Health and Suicide Prevention. Available online: https:/ / www.thetrevorproject.org/2020/04/03/implications-of-covid-19-for-lgbtq-youth-mentalhealth-and-suicide-prevention/ (accessed on 28 July 2021).

9. Ruprecht, M.M.; Wang, X.; Johnson, A.K.; Xu, J.; Felt, D.; Ihenacho, S.; Stonehouse, P.; Curry, C.W.; DeBroux, C.; Costa, D.; et al. Evidence of social and structural COVID-19 disparities by sexual orientation, gender identity, and race/ethnicity in an urban environment. J. Urban Health 2021, 98, 27-40. [CrossRef] [PubMed]

10. Urban Institute; McMorrow, S.; Gonzalez, D.; Caraveo, C.A.; Kenney, G.M. Urgent Action Needed to Address Children's Unmet Health Care Needs during the Pandemic. Available online: https://www.urban.org/sites/default/files/publication/103090 /urgent-action-needed-to-meet-childrens-unmet-health-care-needs_0.pdf (accessed on 28 July 2021).

11. Salerno, J.P.; Devadas, J.; Pease, M.; Nketia, B.; Fish, J.N. Gender and sexual minority stress amid the COVID-19 pandemic: Implications for LGBTQ young persons' mental health and well-being. Public Health Rep. 2020, 135, 721-727. [CrossRef]

12. Jarrett, B.; Peitzmeier, S.M.; Restar, A.; Adamson, T.; Howell, S.; Baral, S.; Beckham, S.W. Gender-affirming care, mental health, and economic stability in the time of COVID-19: A global cross-sectional study of transgender and non-binary people. medrXiv 2020. [CrossRef]

13. van der Miesen, A.I.; Raaijmakers, D.; van de Grift, T.C. “You have to wait a little longer": Transgender (mental) health at risk as a consequence of deferring gender-affirming treatments during COVID-19. Arch. Sex. Behav. 2020, 49, 1395-1399. [CrossRef] [PubMed]

14. Silliman Cohen, R.I.; Bosk, E.A. Vulnerable youth and the COVID-19 pandemic. Pediatrics 2020, 146, e20201306. [CrossRef]

15. Okoro, C.A.; Zhao, G.; Fox, J.B.; Eke, P.I.; Greenlund, K.J.; Town, M. Surveillance for health care access and health services use, adults aged 18-64 years-Behavioral Risk Factor Surveillance System, United States, 2014. MMWR Surveill. Summ. 2017, 66, 1-42. [CrossRef] [PubMed] 
16. Radloff, L.S. The CES-D scale: A Self-report depression scale for research in the general population. Appl. Psychol. Meas. 1977, 1, 385-401. [CrossRef]

17. SAS Institute Inc. SAS System; SAS Publishing: Cary, NC, USA, 2020.

18. StataCorp. Stata Statistical Software Release 14; StataCorp LP: College Station, TX, USA, 2015.

19. Hatchel, T.; Ingram, K.M.; Mintz, S.; Hartley, C.; Valido, A.; Espelage, D.L.; Wyman, P.; Hong, J.S.; Rose, C.A.; Espelage, D.L. Predictors of suicidal ideation and attempts among LGBTQ adolescents: The roles of help-seeking beliefs, peer victimization, depressive symptoms, and drug use. J. Child Fam. Stud. 2019, 28, 2443-2455. [CrossRef]

20. Konnoth, C. Supporting LGBT communities in the COVID-19 pandemic. In Assessing Legal Responses to COVID-19; Burris, S., de Guia, S., Gable, L., Levin, D.E., Parmet, W.E., Terry, N.P., Eds.; Public Health Law Watch: Boston, MA, USA, 2020 ; pp. $234-239$.

21. De France, K.; Evans, G.W. Expanding context in the role of emotion regulation in mental health: How socioeconomic status (SES) and developmental stage matter. Emotion 2020, 21, 772-782. [CrossRef] [PubMed]

22. Agberotimi, S.F.; Akinsola, O.S.; Oguntayo, R.; Olaseni, A.O. Interactions between socioeconomic status and mental health outcomes in the Nigerian context amid COVID-19 pandemic: A comparative study. Front. Psychol. 2020, 11, 559819. [CrossRef] [PubMed]

23. Statistics Canada. Total Income Explorer, 2016 Census. Available online: https://www12.statcan.gc.ca/census-recensement/20 16/dp-pd/dv-vd/inc-rev/index-eng.cfm (accessed on 28 July 2021).

24. Statistics Canada. Highest Level of Educational Attainment (General) by Selected Age Groups 15 Years and Over. Available online: https: / / www12.statcan.gc.ca / census-recensement/2016/dp-pd/hlt-fst/edu-sco/Table.cfm?Lang=E\&T=11\&Geo=00 $\& \mathrm{SP}=1 \&$ view $=2 \&$ age $=1 \&$ sex $=1$ (accessed on 28 July 2021).

25. Seidler, Z.E.; Dawes, A.J.; Rice, S.M.; Oliffe, J.L.; Dhillon, H.M. The role of masculinity in men's help-seeking for depression: A Systematic review. Clin. Psychol. Rev. 2016, 49, 106-118. [CrossRef]

26. Yousaf, O.; Popat, A.; Hunter, M.S.; Levant, R.F. An investigation of masculinity attitudes, gender, and attitudes toward psychological help-seeking. Psychol. Men Masc. 2015, 16, 234-237. [CrossRef]

27. Chan, R.C.; Operario, D.; Mak, W.W.S. Bisexual individuals are at greater risk of poor mental health than lesbians and gay men: The mediating role of sexual identity stress at multiple levels. J. Affect. Disord. 2020, 260, 292-301. [CrossRef]

28. Morley, D.; Street, C. Mixed Experiences: Growing Up Mixed Race Mental Health and Wellbeing; National Children's Bureau: London, $\mathrm{UK}, 2014$.

29. Chu, K.M.; Garcia, S.M.S.; Koka, H.; Wynn, G.H.; Kao, T. Mental health care utilization and stigma in the military: Comparison of Asian Americans to other racial groups. Ethn. Health 2021, 26, 235-250. [CrossRef]

30. Hudson, C.G. Socioeconomic status and mental illness: Tests of the social causation and selection hypothesis. Am. J. Orthopsychiatry 2005, 75, 3-18. [CrossRef]

31. Sasaki, N.; Kuroda, R.; Tsuno, K.; Imamura, K.; Kawakami, N. Deterioration in mental health under repeated COVID-19 outbreaks greatest in the less educated: A cohort study of Japanese employees. J. Epidemiol. 2021, 31, 93-96. [CrossRef] [PubMed]

32. Goldberg, A.E.; Kuvalanka, K.A.; Budge, S.L.; Benz, M.B.; Smith, J.Z. Health care experiences of transgender binary and nonbinary university students. Couns. Psychol. 2019, 47, 59-97. [CrossRef]

33. Ferrie, J.; Miller, H.; Hunter, S.C. Psychosocial outcomes of mental illness stigma in children and adolescents: A mixed-methods systematic review. Child. Youth Serv. Rev. 2020, 113, 104961. [CrossRef]

34. Rogers, M.L.; Hom, M.A.; Janakiraman, R.; Joiner, T.E. Examination of minority stress pathways to suicidal ideation among sexual minority adults: The moderating role of LGBT community connectedness. Psychol. Sex. Orientat. Gend. Divers. 2020, 8 , 38-47. [CrossRef]

35. Smith, J.P.; Randall, C.L. Anxiety and alcohol use disorders: Comorbidity and treatment considerations. Alcohol Res. 2012, 34, 414-431.

36. Lipari, R.N.; Van Horn, S. Smoking and Mental Illness among Adults in the United States. The CBHSQ Report; Substance Abuse and Mental Health Services Administration: Rockville, MD, USA, 2017.

37. Burns, L.; Teesson, M. Alcohol use disorders comorbid with anxiety, depression and drug use disorders: Findings from the Australian National Survey of Mental Health and Well Being. Drug Alcohol Depend. 2002, 68, 299-307. [CrossRef]

38. Wamamili, B.; Wallace-Bell, M.; Richardson, A.; Grace, R.C.; Coope, P. Associations of history of mental illness with smoking and vaping among university students aged 18-24 years in New Zealand: Results of a 2018 national cross-sectional survey. Addict. Behav. 2021, 112, 106635. [CrossRef]

39. Special Advisory Committee on the Epidemic of Opioid Overdoses. Opioids and Stimulant-Related Harms in Canada. Available online: https:/ / health-infobase.canada.ca/substance-related-harms/opioids-stimulants (accessed on 28 July 2021).

40. Salerno, J.P.; Pease, M.; Devadas, J.; Nketia, B.; Fish, J.N. COVID-19-Related Stress among LGBTQ + University Students: Results of a US National Survey. Available online: https://drum.lib.umd.edu/bitstream/handle/1903/26375/LGBTQ_COVID_Report_ 09032020.pdf? sequence $=3 \&$ isAllowed $=y$ (accessed on 28 July 2021).

41. Jessup, M.A. Unmet mental health and substance abuse treatment needs of sexual minority elders. J. Homosex. 2012, 59, 654-674. [CrossRef] [PubMed]

42. Steele, L.S.; Ross, L.E.; Epstein, R.; Strike, C.; Goldfinger, C. Correlates of mental health service use among lesbian, gay, and bisexual mothers and prospective mothers. Women Health 2008, 47, 95-112. [CrossRef] 
43. Canadian Institute for Health Information. Child and Youth Mental Health in Canada [Infographic]. Available online: https: //www.cihi.ca/en/child-and-youth-mental-health-in-canada-infographic (accessed on 21 October 2021).

44. Rose, D.; Ussher, J.; Perz, J. Let's talk about gay sex: Gay and bisexual men's sexual communication with healthcare professionals after prostate cancer. Eur. J. Cancer Care 2017, 26, e12469. [CrossRef] [PubMed] 